Col d pl asma on ful I-thi ckness cut aneous wound accel er at es heal ing through promoting i nf I ammat i on, re-epi thel i al i zat i on and wound cont $r$ act $i$ on

\begin{tabular}{|c|c|}
\hline 著者 & $\begin{array}{l}\text { Nasr uddi n, Nakaj i ma Yukar i, Mukai Kanae, } \\
\text { Rahay u Heni Set yowat i Est i, Nur Muhammad, } \\
\text { I shi j i ma Tat suo, Enomot o Hi r oshi , Uesugi } \\
\text { Yoshi hi ko, Sugama Junko, Nakat ani Toshi o }\end{array}$ \\
\hline $\begin{array}{l}\text { j our nal or } \\
\text { publ i cat } i \text { on } t i t l e\end{array}$ & Cl i ni cal Pl asma Medi ci ne \\
\hline vol une & 2 \\
\hline nunber & 1 \\
\hline page $r$ ange & $28-35$ \\
\hline year & $201407-01$ \\
\hline URL & ht t p: //hdl . handl e. net /2297/36941 \\
\hline
\end{tabular}




\section{Cold plasma on full-thickness cutaneous wound accelerates healing through promoting inflammation, re-epithelialization and wound contraction}

Nasruddin $^{1,2}$,Yukari Nakajima ${ }^{1}$, Kanae Mukai ${ }^{1}$, Heni Setyowati Esti Rahayu ${ }^{2}$, Muhammad Nur ${ }^{3}$, Tatsuo Ishijima ${ }^{4}$, Hiroshi Enomoto ${ }^{4,5}$,Yoshihiko Uesugi ${ }^{4,6}$, Junko Sugama $^{7}$, Toshio Nakatani ${ }^{7}$

${ }^{1}$ Department of Clinical Nursing, Graduate School of Medical Science, Kanazawa University, Kanazawa-shi, Japan

${ }^{2}$ Faculty of Health Science, Universitas Muhammadiyah Magelang, Magelang, Central Java, Indonesia

${ }^{3}$ Department of Physics, Diponegoro University, Semarang, Indonesia

${ }^{4}$ Research Center for Sustainable Energy and Technology, Division of Energy and Environmental Technology, Kanazawa University, Kanazawa-shi, Japan

${ }^{5}$ Faculty of Mechanical Engineering, Institute of Science and Engineering, Kanazawa University, Kanazawa-shi, Japan

${ }^{6}$ Faculty of Electrical and Computer Engineering, Institute of Science and Engineering, Kanazawa University, Kanazawa-shi, Japan

${ }^{7}$ Division of Nursing, Faculty of Health Sciences, Institute of Medical, Pharmaceutical and Health Sciences, Kanazawa University, Kanazawa-shi, Japan 


\section{Corresponding author:}

Prof. Toshio Nakatani

Division of Nursing, Faculty of Health Sciences, Institute of Medical, Pharmaceutical and Health

Sciences, Kanazawa University

5-11-80 Kodatsuno, Kanazawa-shi, Ishikawa-ken 920-0942, Japan

Tel.: +81 762652542

Fax: +81 762344363

Email: nakatosi@staff.kanazawa-u.ac.jp 


\begin{abstract}
We investigated cold plasma effects on acute wounds of mice. The mice were classified into experimental and control groups. In the former, wounds were treated using cold plasma once daily for 1 minute, and then covered with hydrocolloid dressing; wounds in the control were left to heal under hydrocolloid dressing. Daily evaluation was conducted for 15 days. General and specific staining was applied to evaluate re-epithelialization, neutrophil, macrophage, myofibroblast and transforming growth factor beta. It was found that cold plasma accelerated wound healing by one day. Plasma may promote the late phase of inflammation, accelerate reepithelialization and increase wound contraction.
\end{abstract}




\section{INTRODUCTION}

Cutaneous wound healing is a complex physiological process consisting of orchestrated events communicated by collaborative factors ${ }^{1}$. The utilization of various exogenous agents from natural products like Indonesian honey ${ }^{2}$ and oleic or linoleic acid ${ }^{3}$ to physical tools like light ${ }^{4}$ and laser ${ }^{5}$ hasbeen shown to enhance the overlapping healing phases, including inflammation, proliferation and remodeling ${ }^{1}$. Among these, wound therapy based on cold plasma, that is, nonequilibrium plasma (with an electron temperature much higher than the gas temperature), with a low temperature of ionized $\operatorname{gas}^{6}$, has opened the possibility of a paradigm shift in biomedical therapy ${ }^{7}$;it has drawn substantial attention from both plasma and wound care scientists since its feasibility to work through living tissue ${ }^{8,9}$ and its potency for resolving problems in contemporary wound care were demonstrated ${ }^{10}$. As the fourth state of matter ${ }^{6}$, plasma has the ability to produce controllable reactive species, like nitric oxide (NO) and hydroxyl radicals $(\mathrm{OH})$, upon contacting the open air ${ }^{11}$, as well as $\mathrm{OH}$ radicals and hydrogen peroxide $\left(\mathrm{H}_{2} \mathrm{O}_{2}\right)$ upon contacting an aqueous solution ${ }^{12}$. Although the clinical efficacy of carefully controlled treatment with cold plasma for killing bacteria colonizing chronic wounds ${ }^{13,14}$ and improving wound healing ${ }^{15}$ has been demonstrated, there have been few studies about the effects of cold plasma and its mechanism of action on acute wounds in mouse models.

Re-epithelialization and wound contraction are two key events in the healing of fullthickness wounds. The former is central to wound closure, which is closely connected to granulation tissue formation in a spatiotemporal manner ${ }^{16}$, and the latter may account for up to a $40 \%$ decrease in wound size, correlated with the expression of myofibroblasts ${ }^{17}$. It is well established that these processes are influenced by the presence of growth factors like epidermal 
growth factor (EGF), keratinocyte growth factor (KGF) and transforming growth factor (TGF) ${ }^{15}$, which are likely mediated by reactive oxygen species (ROS) and $\mathrm{NO}^{18-20}$.

Although the mechanism of the interaction between cold plasma and cells or living tissue is still unclear ${ }^{21}$, several studies have reported the effects of cold plasma on key wound-related cells or sub-cells, included promoting theproliferationoffibroblasts ${ }^{22}$ and endothelial cells ${ }^{23}$, as well as the growth of epithelial cells ${ }^{24}$, inhibiting the migration of fibroblasts ${ }^{25}$ and their surface expression $^{26}$, and activating integrin of fibroblasts and epithelial cells ${ }^{27}$. Interestingly, some of these effects are likely to be similar to the activities of natural ROS and/or NO during wound healing, particularly cold plasma's effects on the proliferation of both fibroblasts and endothelial cells $^{19}$. Therefore, the aim of this study was to assess the effects of cold plasma on acute cutaneous wound healing in an in vivo scenario with a focus on re-epithelialization and wound contraction.

\section{MATERIALS AND METHODS}

\section{Cold plasma jet characterization and mouse wound positioning}

The cold atmospheric pressure plasma jet system that we used here is similar to the device developed by Teschke et $a l .{ }^{28}$.Two metal ring electrodes were used around the quartz tube for the cold atmospheric pressure plasma jet system provided by the Division of Electrical Engineering and Computer Science, Kanazawa University, Kanazawa, Japan. It had a quartz tube with a 1.6 $\mathrm{mm}$ inner diameter. A low-frequency $(\sim 20 \mathrm{kHz}) \mathrm{AC}$ high voltage, with a peak-to-peak voltage of $25 \mathrm{kV}$, was applied to the two ring electrodes when commercial argon gas (99.995\% purity) at a flow rate of $5 \mathrm{slm}$ was injected from one end of the quartz tube. The discharge voltage and discharge current were measured with a high-voltage probe (P6015A; Tektronix, Inc., Tokyo, 
Japan) and a current probe (8585C; Pearson Electronics, Palo Alto, CA, USA). The average power density at the electrode was $85 \mathrm{~W} / \mathrm{cm}^{2}$ in this study.

During its treatment, a mouse wound was positioned about $15 \mathrm{~mm}$ under the nozzle of the plasma reactor, and was not touched by its jet. Optical emission spectroscopy (OES) measurement at about $10 \mathrm{~mm}$ under the nozzle showed the emissions of the $\mathrm{OH}$ (A-X transition) transition near $309 \mathrm{~nm}, \mathrm{~N}_{2}$ (C-B transition) (band head maximum at $\left.337 \mathrm{~nm}\right)^{29}$ and $\mathrm{Ar} \mathrm{I}$ (maximum $763 \mathrm{~nm}$ ). This observation revealed the presence of both hydroxyl radical $(\mathrm{OH})$ and nitrogen-based reactive species in the gas phase during its generation (Figure 1).

In order to evaluate its thermal effect on living tissue, cold plasma jet was tested on normal skin of anesthetized mouse prior to its application on wound. On the basis of measurement with a noncontact infra-red digital camera (F30S; NEC Avio Infrared Technologies, Tokyo, Japan) at room temperature $\left(24^{\circ} \mathrm{C}\right)$, during cold plasma treatment, the temperature of the influenced skin, of non influenced skin and of the end of the plasma nozzle were $32.3{ }^{\circ} \mathrm{C}, 28.8{ }^{\circ} \mathrm{C}$ and $36.6{ }^{\circ} \mathrm{C}$, respectively. After the session, injury was not observed on treated skin.

\section{Animals and experimental protocol}

Forty BALB/c CrSlc male mice aged 8 weeks (Sankyo Lab Service Corporation, Inc., Toyama, Japan) and weighing 21.3-26.0 g were used. They were caged individually in an air-conditioned room at $25.0 \pm 2.0^{\circ} \mathrm{C}$ with light from $08: 45$ to $20: 45 \mathrm{~h}$. Water and laboratory chow were given freely. The experimental protocol and animal care were in accordance with the Guidelines for the Care and Use of Laboratory Animals of Kanazawa University, Japan (AP: 112243). 


\section{Wound healing model and plasma treatment}

After being completely anesthetized by the injection of pentobarbital sodium $(0.5 \mathrm{mg} / 10 \mathrm{~g}$ weight $)$ into the peritoneal cavity, we held the skin of the dorsum including the subcutaneous tissue between thumb and finger, folded it along the apex of the median line on the dorsum in a Ushape, put both sides of the skin together, made two holes through the skin with a sterile disposable $2 \mathrm{~mm}$ biopsy punch (Kai Industries Co. Ltd., Gifu, Japan) and finally made two circular (2 $\mathrm{mm}$ in diameter) full-thickness skin wounds including the panniculus carnosus muscle and part of the subcutaneous tissue on both sides of the dorsum of the mouse. Subsequently, the mice were randomly classified into two groups: (1) Experimental group, with wounds treated once daily by a cold plasma jet during 1 minute in one spot on the wound, and then covered by hydrocolloid dressing (Tegaderm; 3M Health Care, Tokyo, Japan) to maintain its moist environment; and (2) Control group, with wounds only allowed to heal under hydrocolloid dressing.

\section{Macroscopic evaluation}

The day when wounds were made was designated as day 0 , and the process of wound healing was observed daily from days 0 to 15 after wounding. Before observation, the surrounding environment of wounds was cleaned with saline solution. Wounded edges were traced on polypropylene sheets and photographs were taken every day. The traces on the sheets were captured with a scanner onto a personal computer using Adobe Photoshop Elements 7.0 (Adobe System Inc., Tokyo, Japan), and the areas of wounds were calculated using image analysis software Scion Image Beta 4.02 (Scion Corporation, Frederick, Maryland, USA). 


\section{Calculation of healing day}

The day of wound healing was calculated based on a graph of the ratios of areas to original areas. Initially, the overall trend of such a graph was evaluated. Wound healing day was plotted on the $\mathrm{y}$-axis when the trend of reduction of wound size started to become flat, which was at 0.15 .

\section{Histology}

The mice were euthanized by a massive pentobarbital sodium IP injection on day 3, 7, 11 or 15 post-wounding. The wounds and the surrounding normal skin were excised for an area of about $10 \mathrm{~mm} \times 10 \mathrm{~mm}$ square, stapled onto polypropylene sheets to prevent over-contraction of the samples and fixed in neutral buffered $10 \%$ formalin solution in $0.01 \mathrm{M}$ phosphate buffer, $\mathrm{pH} 7.4$, for about 15 hours. The samples were then rinsed in $0.01 \mathrm{M}$ phosphate-buffered saline (PBS) for about 8 hours. Subsequently, they were dehydrated in an alcohol series, cleaned in xylene and embedded in paraffin to prepare serial 5 - $\mu \mathrm{m}$ sections. Next, the sections were separated into two groups: for general staining using hematoxylin-eosin (H\&E) for re-epithelialization observation, and for specific staining using immunohistochemical staining.

\section{Immunohistochemistry (IHC)}

Immunohistochemical staining for myofibroblasts was conducted for the sections from all days of mouse harvesting (days3, 7, 11 and 15), while that for macrophages, neutrophils and transforming growth factor beta (TGF- $\beta$ ) was only conducted for the sections from days 3 and 7 
of mouse harvesting because of a limitation in the number of sections. In brief, the immunohistochemical staining was performed as follows. After deparaffinization and rehydration, antigen unmasking was accomplished by heating slides in a water bath followed by incubation in sodium citrate buffer $(10 \mathrm{mM}$ sodium citrate, $0.05 \%$ Tween $20, \mathrm{pH} 6.0)$ for 20 minutes at approximately $100{ }^{\circ} \mathrm{C}$. Then, the slides were washed with PBS, pH 7.4, covered with $0.03 \%$ hydrogen peroxide to block endogenous peroxidase for 5 minutes at room temperature, rinsed with distilled water, covered with protein-free normal serum for 10 minutes at room temperature and then rinsed with PBS. Subsequently, sections were incubated with primary and secondary antibodies as follows:

a. For neutrophil identification

Sections were incubated with anti-neutrophil antibody (Abcam Japan, Tokyo, Japan)at a dilution of 1:100 in PBS at $4{ }^{\circ} \mathrm{C}$ overnight, and then with secondary antibody polyclonal rabbit anti-rat immunoglobulins/HRP (Dako, North America Inc., CA) + mouse serum (Dako North America Inc., CA)(1:100 in PBS) at room temperature for 30 minutes.

b. For macrophage identification

Sections were incubated with anti-macrophage-3 antibody (Abcam Japan, Tokyo, Japan)at a dilution of 1:100 in PBS at $4{ }^{\circ} \mathrm{C}$ overnight and then with secondary antibody polyclonal rabbit anti-rat immunoglobulins/HRP (Dako, North America Inc., CA) + mouse serum (Dako North America Inc., CA)(1:100 in PBS) at room temperature for 30 minutes.

c. For myofibroblast identification

Sections were incubated with anti- $\alpha$-smooth muscle actin (anti- $\alpha$-SMA) (Abcam Japan, Tokyo, Japan) (1:100 in Tween-PBS) at $4^{\circ} \mathrm{C}$ overnight, and then with secondary antibody Dako 
EnVision+System-HRP Labeled Polymer Anti-Mouse (Dako North America Inc., CA) at room temperature for 30 minutes.

d. For TGF- $\beta$ identification

Sections were incubated with anti-TGF- $\beta$ (1:100 in PBS) at $4{ }^{\circ} \mathrm{C}$ overnight, and then with secondary antibody Dako EnVision+System-HRP Labeled Polymer Anti-Mouse (Dako North America Inc., CA)at room temperature for 30 minutes.

After completion of the incubation with the secondary antibody, the sections were reacted with 3,3'-diaminobenzidine substrate (Dako ENVISION Kit/HRP (DAB), Dako Japan, Kyoto, Japan) for staining for about 2-5 minutes at room temperature. Finally, counterstaining was conducted using hematoxylin. As negative controls, samples were prepared using the same procedure with PBS instead of the first antibody.

\section{Microscopic observations}

On the basis of the results of hematoxylin-eosin staining, the percentage of re-epithelialization was calculated as follows: $100 \% \mathrm{x}$ (length of new epithelium/length of wound between wound edges). In addition, on the basis of the results of immunohistochemical staining, the numbers of neutrophils, macrophages, myofibroblasts and cells stained with TGF- $\beta$ through observation using an Olympus BX50 light microscope (Olympus, Tokyo, Japan) at magnification 400x were also counted. Images were captured with Olympus DP72 digital camera and Olympus DP2-BSW software. Three squares were selected at each wound margin and the center of the wound, on four serial sections per wound. The data are presented as the mean number of stained cells counted in the twelve squares; four serial wound sections per wound were analyzed. 


\section{Statistical analysis}

Data were subjected to statistical analyses using SPSS 16.0. Differences between the experimental and control groups for the ratio of wound area average to original wound area and the calculation of healing day were evaluated by two-tailed unpaired t-tests and $\mathrm{p}$-values $<0.05$ were considered significant. Differences between the experimental and control groups for the results of histological staining were evaluated by ANOVA followed by Tukey test and p-values $<0.05$ were considered significant.

\section{RESULTS}

\section{Macroscopic evaluation}

Immediately after cold plasma irradiation, the wound surfaces in the experimental group seemed drier than those in the control group. Wounds were observed daily (Figure 2). There were no particular differences regarding the appearance of the wound surfaces between the experimental and control groups during this observation. Apparent differences just in the wound size were found for several days of this observation period.

Wounds in both the experimental and the control groups experienced slight expansion (edema) on day 1 and then decreased gradually until the end of the observation period. On days 1 to 3 , there was exudate on the surface of the wounds in the two groups. The wound area of the experimental group was smaller than that of the control from days 4 to 15 . From days 5 to 15 , the surfaces of the wounds in all groups were mostly fresh with no exudate. 


\section{Wound area evaluation and day of wound healing}

The ratios of wound areas to original areas from day 0 both until day 15 and until the day of wound healing were determined (Figure 3a). On days 3, 4, 5, 6 and 8, the ratios of areas to the original area for the experimental group were significantly lower than those for the control group $(P<0.05)$. On the other hand, on days 9 to 15 , the ratios of areas to the original area for the experimental group were mostly the same as those for the control group $(P>0.05)$. Days of wound healing for the experimental and control groups were $8.0 \pm 0.6$ and $9.0 \pm 1.3$, respectively (Figure 3b). These two means were significantly different $(P=0.04)$.

\section{Cold plasma accelerated re-epithelialization}

Re-epithelialization during wound healing was observed (Figure 4). On day 3, the percentages of re-epithelialization were significantly different between the experimental group and the control group $(P<0.001)$, with the former being more than $25 \%$ greater than the latter. The percentages of re-epithelialization of both increased dramatically from days 3 to 7 (Control group: $P<0.001$; Experimental group: $P<0.001)$. On day 7, the percentages of re-epithelialization of the two groups were similar $(P=0.999)$. On days 11 and 15 , all wounds in all groups were covered by new epithelium.

\section{Myofibroblast count}

Myofibroblast number on day 3 was evaluated. On this day, a few myofibroblasts were observed in the experimental group. On the other hand, no myofibroblasts were observed in the control. Myo fibroblasts were counted on days 7,11 and 15 (Figure 5). The number of myofibroblasts per

$\mathrm{mm}^{2}$ in the experimental group peaked on day 7 and then decreased gradually until day 15 , while 
that in the control was stable on days 7 and 11 and then decreased on day 15 . On day 7 , the number of myofibroblasts in the experimental group was higher than that in the control group, but the two means were not significantly different $(P=0.557)$. On the other hand, on days 11 and 15 , the numbers of myofibroblasts in the experimental group were slightly lower than those in the control, but the two means were not significantly different ( 11 days: $P=0.990$, 15 days: 0.994). On day 15, the numbers of myofibroblasts in the experimental group and the control were lower than those on day 7. The two means in the former group were significantly different $(P<0.001)$, but those in the latter were not $(P=0.148)$.

\section{Neutrophil count}

Numerous neutrophils were observed in the experimental group and the control group on days 3 and 7 after wounding (Figure 6). On day 3, the numbers of neutrophils per $\mathrm{mm}^{2}$ in the experimental and control groups were relatively similar and were not significantly different $(P=0.966)$. On day 7 , the number of neutrophils in the experimental group was lower than that in the control group, but the two means were not significantly different $(P=0.762)$. The number of neutrophils in the two groups decreased rapidly from day 3 to day 7 (Experimental group: $P<0.05$; Control group: $P<0.05)$.

\section{Macrophage count}

Numerous macrophages in the experimental group and the control group were observed on days 3 and 7 after wounding (Figure 7). On these different observation days, the macrophage number

per $\mathrm{mm}^{2}$ in the experimental group was lower than that in the control, but the two means were not significantly different (day 3: $P=0.203$; day 7: $P=0.676$ ). Macrophage numbers in the control 
group decreased significantly from day 3 to day $7(P<0.05)$. Macrophage number in the experimental group also decreased from day 3 to day 7, but the two means were not significantly different $(P=0.913)$.

\section{Count of cells stained with TGF-beta}

Numerous cells stained with transforming growth factor beta (TGF- $\beta$ ) were observed in the experimental group and the control group on days 3 and 7 after wounding (Figure 8). On day 3, the number of cells with TGF- $\beta$ per $\mathrm{mm}^{2}$ in the experimental group was lower than that in the control group, but the two means were not significantly different $(P=0.688)$. In contrast, on day 7 , the number of cells with TGF- $\beta$ in the experimental group was slightly higher than that in the control group, but the two means were not significantly different $(P=0.897)$. The numbers of cells with TGF- $\beta$ in the experimental group were similar on day 3 and day $7(P=0.991)$. On the other hand, the number of cells with TGF- $\beta$ in the control group decreased from day 3 to day 7 , but the two means were not significantly different $(P=0.209)$.

\section{DISCUSSION}

This research design separated between treated and untreated mice because it was considered that cold plasma produced not only reactive species like nitric oxide (NO) and hydrogen peroxide $\left(\mathrm{H}_{2} \mathrm{O}_{2}\right)$ that in appropriate dosage may have efficacy for wound healing ${ }^{20}$, but also that temperature change may have the same effect ${ }^{30}$. While the former may work in a locally specific manner ${ }^{25}$, the latter may operate at a physiologically systematic level under a hypothalamic regime ${ }^{31}$. In this design, there were two wounds in a mouse, on the left side and the 
right side. When the left wound was subjected to plasma treatment, the right wound may also have been influenced by its warmth. Therefore, it was important to ensure that there was no influence of plasma agents in the untreated mice. Although this approach was intended to mimic the clinical setting in a hospital, it differed from the work of Heinlin et al. ${ }^{15}$, who placed two in the same patient.

On the basis of histological data from 3 days after wounding, we showed that cold plasma is efficacious for the acceleration of wound re-epithelialization. This finding seemed to be in line with Nastuta et al. and Heinlin et al. Nastuta et al. reported that helium cold plasma treatment accelerated the re-epithelialization of burn wound tissue ${ }^{32}$. Furthermore, by an investigation of clinical standardized photographs of wounds on a skin graft donor site, Heinlin et al. reported that argon cold plasma treatment had a positive effect ${ }^{15}$. However, there were no histological data in these two reports. This is the first report describing such findings on this topic supported by histological data.

In the present study, we found that cold plasma treatment caused acceleration of wound healing by one day compared with that of untreated wounds. Such reduction in the period required for wound healing may be correlated with the early presence and the peak of myofibroblasts in the cold plasma-treated wounds by days 3 and 7 after wound creation, as we observed. It is well established that there are multiple ways by which myofibroblasts originate, one of which is through the differentiation from fibroblasts to myofibroblasts mediated by activated transforming growth factor beta (TGF- $\beta)^{33}$. Cold plasma may play two main roles in influencing this mechanism: (1) promoting the proliferation of fibroblasts on the wound surface, in line with another reported study ${ }^{21}$ in which the higher the number of fibroblasts, the possibly higher the number of myofibroblasts; and (2) activating "latent TGF- $\beta$ " on the wound surface to 
become "active TGF- $\beta$ ", which may increase myofibroblasts. The possibility of cold plasma activating TGF- $\beta$ may involve one or both of two possible mechanisms as follows. The first of these is ROS-based activation, as described previously $y^{34,35}$. ROS in this context are generated by cold plasma. Secondly, it could involve integrin-based activation by cold plasma, as also reported elsewhere ${ }^{26}$.

The activation of TGF- $\beta$ may be detected by an increase of the cells stained with antiTGF- $\beta$ antibody, but in this research, we found no significant difference in the number of cells with TGF- $\beta$ between treated and untreated wounds on days 3 and 7 after wounding. This may be in line with a report mentioned previously that stated that the conventional histological method, as used in this research, could not clearly differentiate between latent TGF- $\beta$ and active TGF$\beta^{36}$.Thus, it is difficult to determine whether immunohistochemical staining of TGF- $\beta$, as in this research, was associated with latent TGF- $\beta$, active TGF- $\beta$, or both.

In the healing of normal wounds, inflammation is classified into early and late phases. Neutrophil-rich and mononuclear-cell-rich infiltrates are representative of the former and the latter, respectively ${ }^{1}$. In this research, the observation that by 3 days after wound creation, there was the same number of neutrophils in the experimental and control groups suggested that there was no difference in the time course of the early phase of inflammation between them. It showed that cold plasma had no effect on the early phase of inflammation. At a later stage, the observation in this research that by 7 days after injury, there were fewer macrophages and neutrophils in the experimental group than in the control suggested that there was an acceleration of the inflammatory phase of repair in the experimental group, so that the late phase of inflammation ended rapidly. So far, it has been revealed that cold plasma treatment accelerated such a process. It has also been indicated that treated wounds started the proliferation phase 
earlier than untreated ones. This phenomenon may be correlated with the proliferative effect of cold plasma, as reported previously ${ }^{22}$, and the early presence of myofibroblasts, as reported in this research.

It is well known that the outcome of acute inflammation is elimination of the noxious stimulus $^{37}$. In this context, Shekhter et al. discussed the possible influence of plasma-based NO on the phagocytosis of macrophages to improve the regeneration of wounds ${ }^{38}$. Theoretically, acute inflammation has two major components: vascular changes and cellular events ${ }^{37}$. Regarding the former, plasma may play the important role of influencing vascular growth, vascular dilation, and microcirculation normalization, as discussed previously ${ }^{38}$. Regarding the latter, the direct effect of cold plasma on the events of inflammatory cells like macrophages has been unclear, but as we wrote previously, it was reported that cold plasma reduced the migration of fibroblasts ${ }^{25}$ and activated their integrin ${ }^{27}$. Migration and integrin activation are crucial events for family members of leukocytes during the inflammatory phase. If the effects of cold plasma on fibroblasts and those on inflammatory cells are the same, this could be used to demonstrate the mechanism of the healing effect of cold plasma during the late stage of the inflammatory phase.

Cold plasma treatment is characteristically topical, with the maximum penetration depth of its reactive oxygen species (ROS)/reactive nitrogen species (RNS) being at most a few tens of micrometers ${ }^{39}$. From a cellular study, it was also reported that the effects of cold plasma were confined to the area that can be reached by ROS/RNS ${ }^{25}$. In the present research, it is unclear whether reactive species of a cold plasma jet could directly reach cells related to wound contraction, myofibroblasts and fibroblasts. However, if this could not be achieved, the hypothesis of Heinlin et al. should be considered. Heinlin et al. hypothesized that the effect of 
cold plasma treatment would probably occur not only in the treatment area, but also in the adjacent wound area through microenvironmental modification ${ }^{15}$.

In this research, we did not detect reactive species of cold plasma at a distance of $15 \mathrm{~mm}$ under the nozzle using an OES spectrophotometer, but we detected slight temperature change on normal skin of anesthetized mice at such a distance using an infrared thermal camera. Of course, the detection of such reactive species could be achieved by other methods, but the slight temperature change should also be considered if a microenvironmental perspective were applied.

In conclusion, it was determined that cold plasma accelerated wound healing by one day through the modification of re-epithelialization, the late stage of inflammation and wound contraction. Cold plasma may influence the wound healing mechanism at the microenvironmental level through not only its reactive species, but also its warming effect, simultaneously.

\section{ACKNOWLEDGMENTS}

Nasruddin would like to acknowledge the help of the Directorate General of Higher Education (DIKTI), Indonesia, which supported her financially during her $\mathrm{PhD}$ study through the Joint Scholarship Program DIKTI, Indonesia-Kanazawa University, Japan.

Part of this work was supported by Grants-in-Aid for Scientific Research, Japan (no. 22592363 and 25293430) and a Grant from The Mitani Foundation for Research and Development.

Conflict of Interest: The authors have no conflicts of interest to report in regard to this manuscript. 


\section{REFERENCES}

1. Clark RAF, Denver MD. Cutaneous tissue repair: Basic biologic considerations. I. Journal of the American Academy of Dermatology. 1985; 13:701-25.

2. Haryanto, Urai T, Mukai K, Suriadi, Sugama J, Nakatani T. Effectiveness of Indonesian honey on the acceleration of cutaneous wound healing: an experimental study in mice. Wounds. 2012; 24:110-9.

3. Rodrigues HG, Vinolo MAR, Magdalon J, Vitzel K, Nachbar RT, Pessoa AFM, dos Santos MF, Hatanaka E, Calder PC, Curi R. Oral administration of oleic or linoleic acid accelerates the inflammatory phase of wound healing. Journal of Investigative Dermatology. 2011; 132:208-215.

4. Fushimi T, Inui S, Nakajima T, Ogasawara M, Hosokawa K, Itami S. Green light emitting diodes accelerate wound healing: characterization of the effect and its molecular basis in vitro and in vivo. Wound Repair Regeneration. 2012; 20: 226-235.

5. Mester E, Spiry T, Szende B, Tota JG. Effect of laser rays on wound healing. The American Journal of Surgery.1971;122:532-535.

6. Fridman G, Friedman G, Gutsol A, Shekhter AB, Vasilets VN, Fridman A. Applied Plasma Medicine. Plasma Processes and Polymers 2008;5:503-33.

7. Keidar M, Walk R, Shashurin A, Srinivasan P, Sandler A, Dasgupta S, Ravi R, Dasgupta, Guerra-Preston R, Trink Bl. Cold plasma selectivity and the possibility of a paradigm shift in cancer therapy. British Journal of Cancer 2011;105:1295-301.

8. Stoffels E. "Tissue Processing" with Atmospheric Plasmas. Contributions to Plasma Physics. 2007;47:40-8.

9. Laroussi M. Low-Temperature Plasmas for Medicine ? IEEE Transaction on Plasma Science. 2009;37 (6):714-25.

10. Lloyd G, Friedman G, Jafri S, Schultz G, Fridman A, Harding K. Gas Plasma: Medical Uses and Developments in Wound Care. Plasma Processes Polymers. 2010;7:194-211.

11. Yonemori S, Nakagawa Y, Ono R, Oda T. Measurement of OH density and air-helium mixture ratio in an atmospheric-pressure helium plasma jet. Journal of Physics D: Applied Physics 2012; 45: 225202. 
12. Ishijima $T$, Hotta $H$, Sugai $H$, Sato M. Multibubble plasma production and solvent decomposition in water by slot-excited microwave discharge. Applied Physics Letters 2007; 91: 121501.

13. Isbary G, Morfill G, Schmidt HU, Georgi M, Ramrath K, Heinlin J, Karrer S, Landthaler M, Shimizu T, Steffes B, Bunk W, Monetti R, Zimmermann J L, Pompl R, Stolz, W. A first prospective randomized controlled trial to decrease bacterial load using cold atmospheric argon plasma on chronic wounds in patients. British Journal of Dermatology. $2010 ; 163: 78-82$.

14. Isbary G, Heinlin J, Shimizu T, Zimmermann JL, Morfill G, Schmidt H-U, Monetti R, Steffes B, Bunk W, Li Y, Klaempfl T, Karrer S, Landthaler M, Stolz W. Successful and safe use of 2 min cold atmospheric argon plasma in chronic wounds: results of a randomized controlled trial. British Journal of Dermatology. 2012;167:404-10.

15. Heinlin J, Zimmermann JL, Zeman F, Bunk W, Isbary G, Landthaler M, Maisch T, Monetti R, Morfill G, Shimizu T, Steinbauer J, Stolz W, Karrer S. Randomized placebocontrolled human pilot study of cold atmospheric argon plasma on skin graft donor sites. Wound Repair and Regeneration 2013; 21: 800-807.

16. Singer AJ, Clark RA. Cutaneous Wound Healing. New England Journal of Medicine. 1999; 341: 738-46.

17. Ai T, Nakatani T, Sugama J, Sanada H, Kitagawa A, Tanaka S. Histological examination of the distribution change of myofibroblast in wound contraction. EWMA 2004; 4:13-20.

18. Stallmeyer B, Kämpfer H, Kolb N, Pfeilschifter J, Frank S. The function of nitric oxide in wound repair: inhibition of inducible nitric oxide-synthase severely impairs wound reepithelialization. Journal of Investigative Dermatology 1999;113:1090-8.

19. Witte MB, Barbul A. Role of nitric oxide in wound repair. The American Journal of Surgery. 2002; 183:406-12.

20. Soneja A, Drews M, Malinski T. Role of nitric oxide, nitroxidative and oxidative stress in wound healing. Pharmacological Reports 2005; 57 Suppl:108-19.

21. Fridman A, Gary F. Plasma Medicine. West Sussex: John Wiley and Sons, 2013.

22. Tipa RS., E.Stoffels. Effects of plasma treatment on wounds. Proceedings of 13th International Conference on Biomedical Engineering (ICBME2008); 2008 Dec 3-6; Singapore: 2009 : 1385-1388. 
23. Kalghatgi S, Friedman G, Fridman A, Clyne AM. Endothelial cell proliferation is enhanced by low dose non-thermal plasma through fibroblast growth factor- 2 release. Annals of Biomedical Engineering 2010; 38:748-57.

24. Hoentsch M, Von Woedtke T, Weltmann K-D, Barbara Nebe J. Time-dependent effects of low-temperature atmospheric-pressure argon plasma on epithelial cell attachment, viability and tight junction formation in vitro. Journal of Physics D: Applied Physics 2012; 45:025206.

25. Volotskova O, Shashurin A, Stepp MA, Pal-Ghosh S, Keidar M. Plasma-controlled cell migration: localization of cold plasma-cell interaction region. Plasma Medicine 2011; 1:85-92.

26. Shashurin A, Stepp M A, Hawley T S, Pal-Ghosh S, Brieda L, Bronnikov S, Jurjus R A and Keidar M. Influence of cold plasma atmospheric jet on surface integrin expression of living cells Plasma Processes and Polymers 2010; 7 : 294-300.

27. Volotskova O, Stepp MA, Keidar M. Integrin activation by a cold atmospheric plasma jet. New Journal of Physics 2012; 14:053019.

28. Teschke M, Kedzierski J, Finantu-Dinu E, Korzec D, Engemann J. High-Speed Photographs of a Dielectric Barrier Atmospheric Pressure Plasma Jet. IEEE Transactions Plasma Science 2005; 33: 310-311.

29. Tsuji M, Kumagae J, Nakano K, Matsuzaki T, Tsuji T. Decomposition of $\mathrm{N}_{2} \mathrm{O}$ in a microwave-absorbent assisted discharge of $\mathrm{N}_{2}$ at atmospheric pressure. Applied Surface Science 2003; 217: 134-148.

30. Xia Z, Sato A, Hughes MA, Cherry GW. Stimulation of fibroblast growth in vitro by intermittent radiant warming. Wound Repair and Regeneration 2000; 8:138-144.

31. Charkoudian N, Skin Blood Flow in Adult Human Thermoregulation: How It Works, When It Does Not, and Why. Mayo Clinical Proceeding 2003; 78: 603-612

32. Nastuta AV, Topala I, Grigoras C, Pohoata V, Popa G. Stimulation of wound healing by helium atmospheric pressure plasma treatment. Journal of Physics D: Applied Physics 2011;44:105204.

33. Hinz B, Phan SH, Thannickal VJ, Galli A, Bochaton-Piallat M-L, Gabbiani G. The myofibroblast: one function, multiple origins. The American Journal of Pathology 2007; 170:1807-16. 
34. Barcellos-Hoff MH, A.Dix T. Redox-mediated activation of latent transforming growth factor-beta1. Molecular Endocrinology 1996; 10:1077-83.

35. Jobling MF, Mott JD, Finnegan MT, Jurukovski V, Erickson AC, Walian PJ, Taylor SE, Ledbetter S, Lawrence CM, Rifkin DB, Barcellos-Hoff MH. Isoform-specific activation of latent transforming growth factor beta (LTGF-beta) by reactive oxygen species. Radiation Research 2006; 166:839-48.

36. Barcellos-Hoff MH, Derynck R, Tsang ML-S, Weatherbee JA. Transforming growth factor-beta activation in irradiated murine mammary gland. Journal of Clinical Investigation 1994; 93:892-909.

37. Kumar BV, Abbas AK, Fausto N, Mitchell R. Acute and Chronic Inflammation. In: Kumar BV, Abbas AK, Fausto N, Mitchell R, editors. Robbins basic pathology,8th edition. Philadelphia: Saunders 2007: 33-43.

38. Shekhter AB, Serezhenkov VA, Rudenko TG, Pekshev AV, Vanin AF. Beneficial effect of gaseous nitric oxide on the healing of skin wounds. Nitric Oxide 2005; 12 : 2010-9.

39. Kong MG, Keidar M, Ostrikov K. Plasmas meet nanoparticles-where synergies can advance the frontier of medicine. Journal of Physics D: Applied Physics 2011; 44: 174018. 


\section{Figure Legends}

Figure 1. Optical emission spectroscopy (OES) measurement of cold plasma jet near the wound surface (about $10 \mathrm{~mm}$ under the nozzle of the cold plasma reactor) during treatment. $\mathrm{OH}$ and nitrogen-based reactive species were identified.

Figure 2.Macroscopic observation of wound healing.

Figure 3: a. Ratio of areas to original areas during wound healing. On days 3,4,5,6 and 8, the treated wound was significantly smaller than that of the control. b. Day of wound healing. Experimental wounds healed significantly faster, by one day, than those of the control.

Figure 4.(a) Percentage of re-epithelialization during wound healing. b: New epithelial image on day 3 of wound healing: (1 and 3) HE staining of the experimental group. (2 and 4) HE staining of the control. $\mathrm{AB}, \mathrm{CD}, \mathrm{EF}$ and $\mathrm{GH}$ show the lengths of new epithelium. IJ and KL also reveal the lengths of new epithelium at magnification 200X. Re-epithelialization increased more rapidly in the experimental group than in the control.

Figure 5. a: Histogram of myofibroblast number on days 7, 11 and 15. By day 7, myofibroblast number in the experimental group was greater than that in the control, but by day 15 , the former was lower than the latter. b: Alpha-SMA staining on days 7 and 15. Black arrows show myofibroblasts colored brown.

Figure 6. a: Histogram for neutrophil number on days 3 and 7 . Neutrophil number of the experimental group was significantly lower than that of the control at day $7 . b$ : Immunohistochemical staining for neutrophils. Black arrows show neutrophils colored brown. 
Figure 7. a: Histogram for macrophage number on days3 and 7. Macrophage number of the experimental group was significantly lower than that of the control on days 3 and 7.b: Immunohistochemical staining for macrophages. Black arrows show macrophages colored brown.

Figure 8. Histogram for the number of cells stained with anti-TGF- $\beta$ antibody on days 3 and 7. 


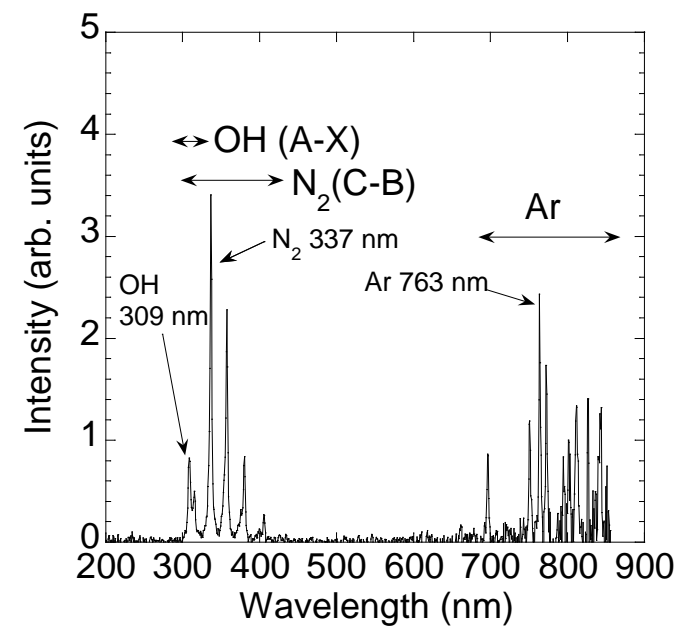

Figure1 
$\begin{array}{lllll}\text { Day } 0 & \text { Day } 3 & \text { Day } 7 & \text { Day } 11 & \text { Day } 15\end{array}$

Control

group

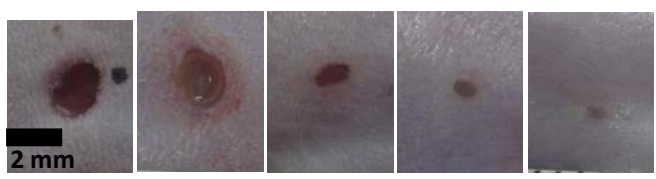

Experimental group

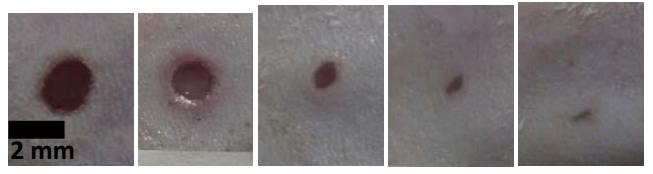

Figure 2

a

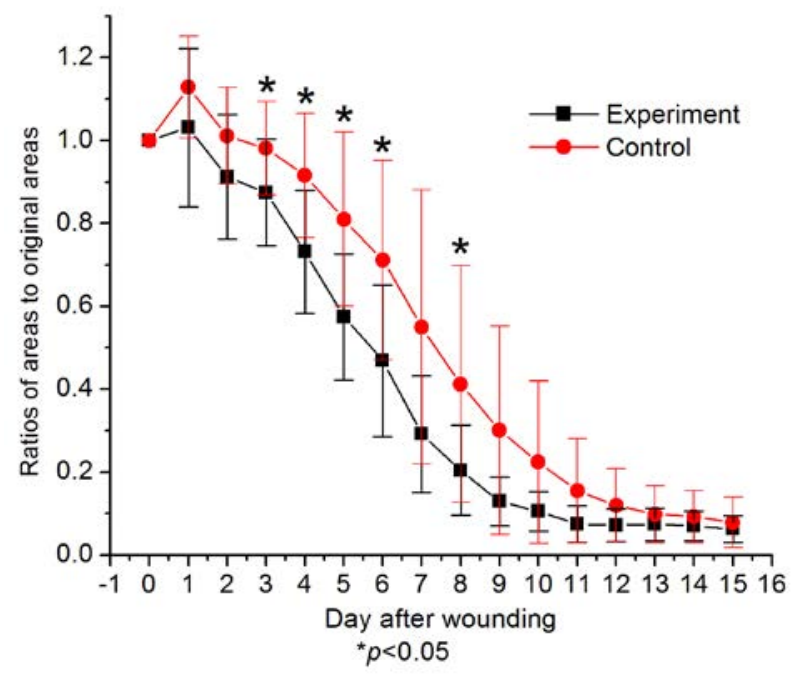

b

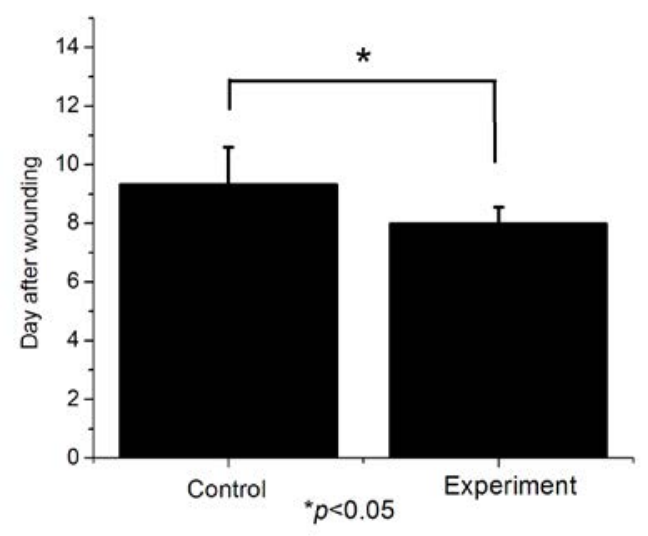

Figure 3 
a

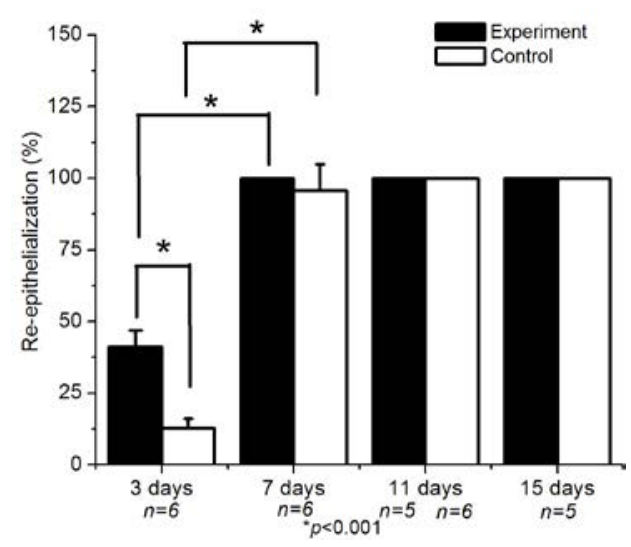

b
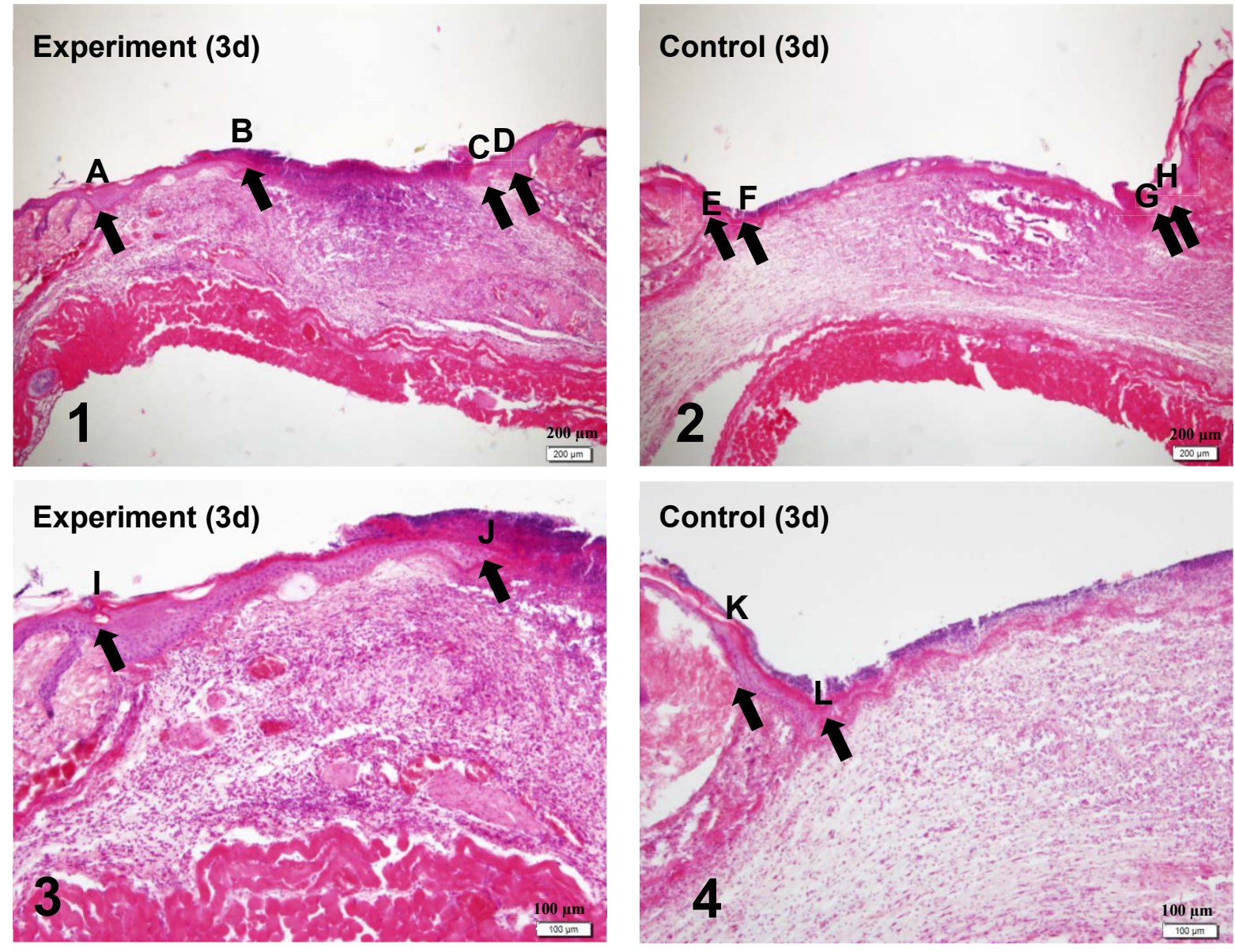

\section{Control (3d)}

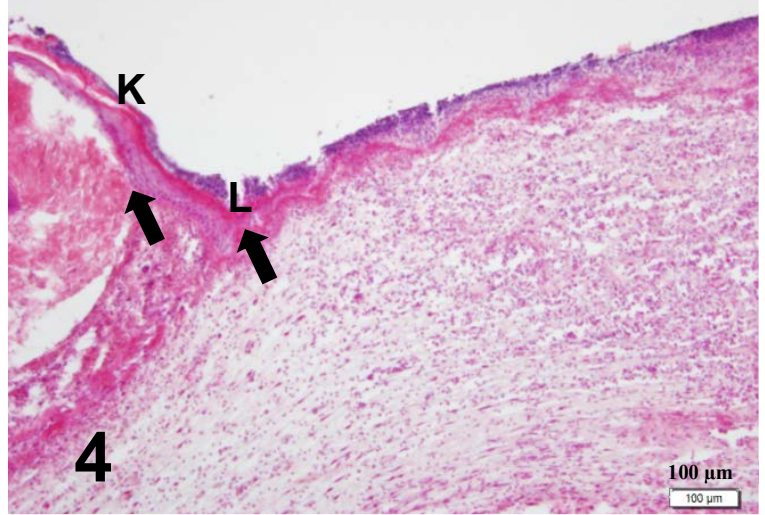

Figure 4. 

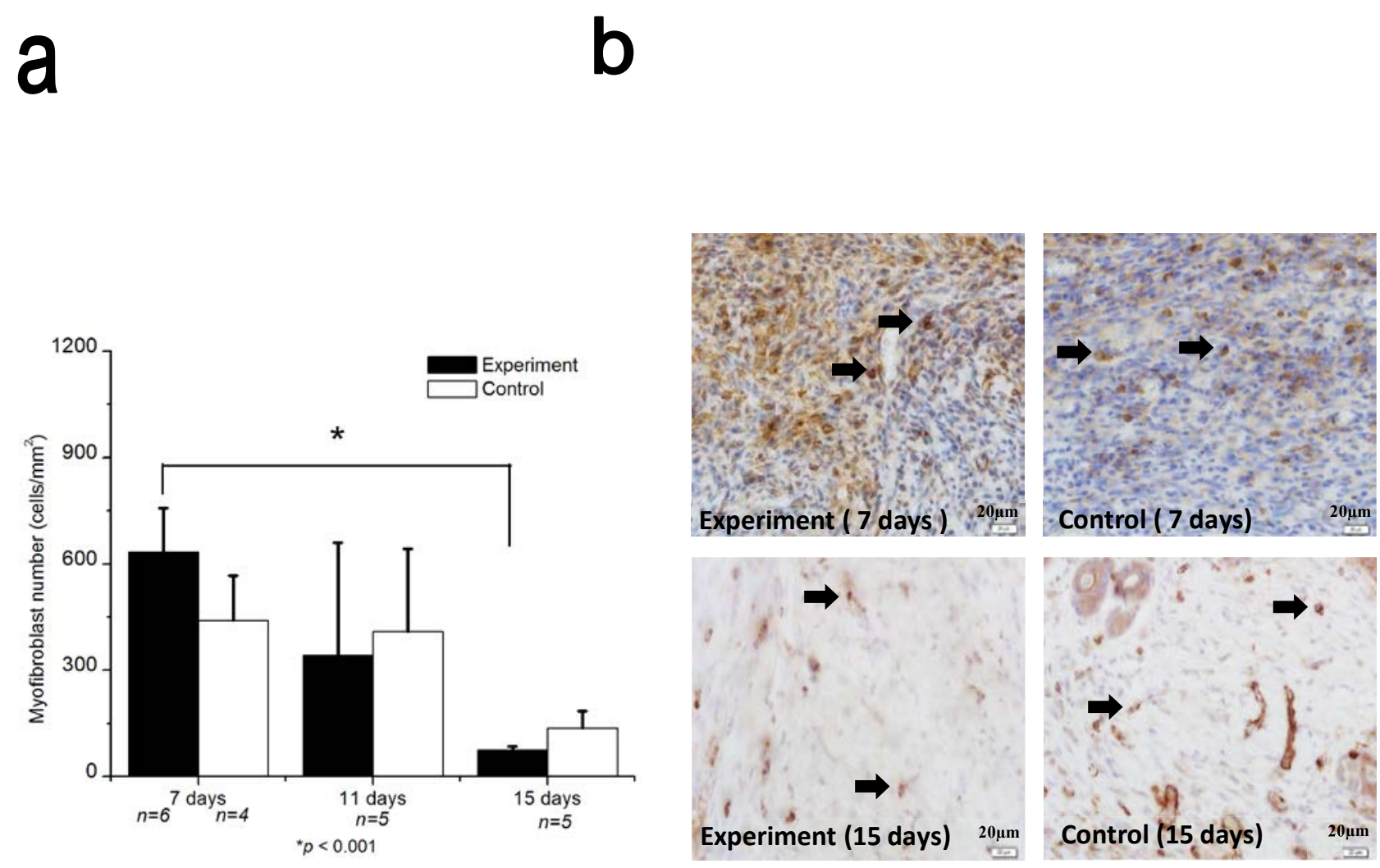

Figure 5. 


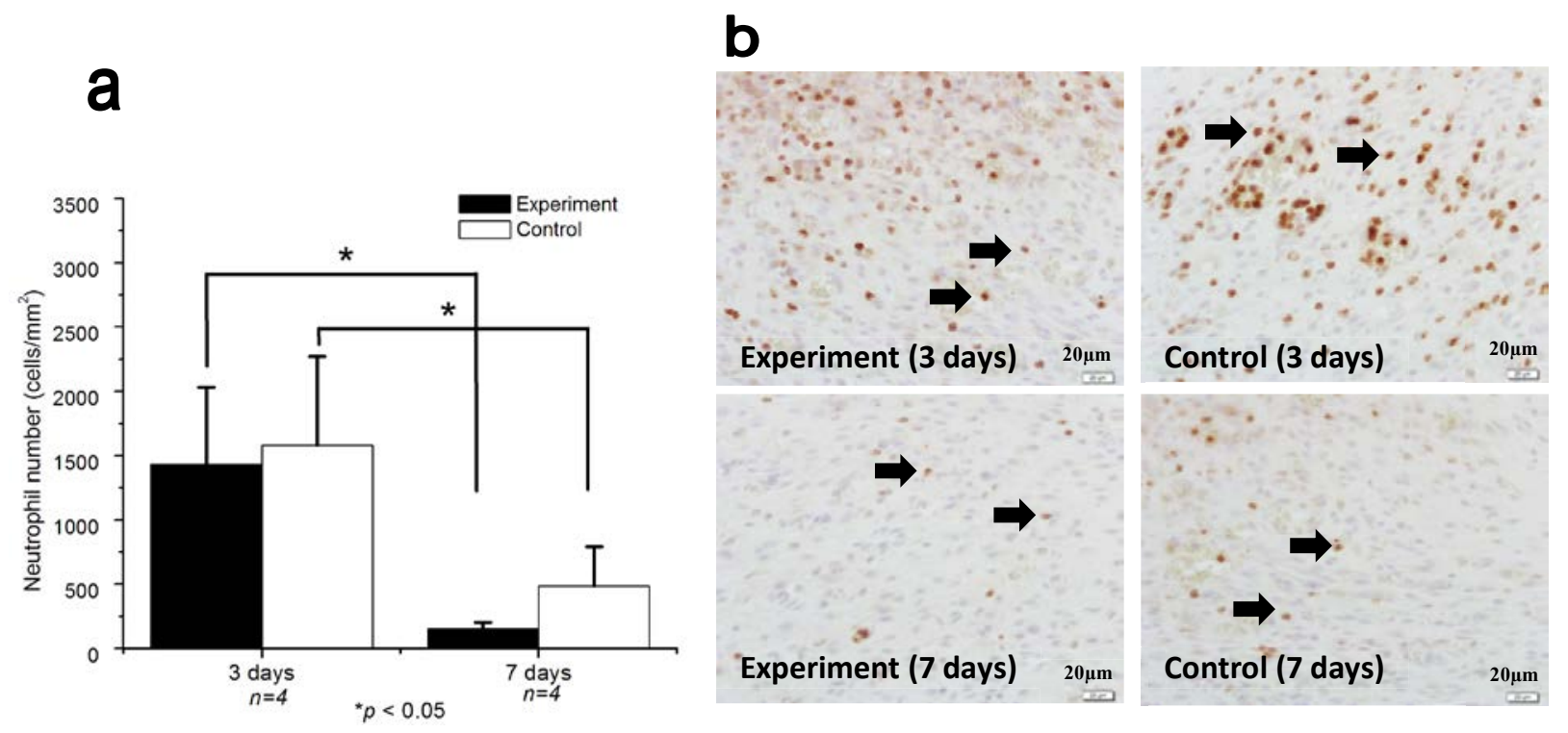

Figure 6

b
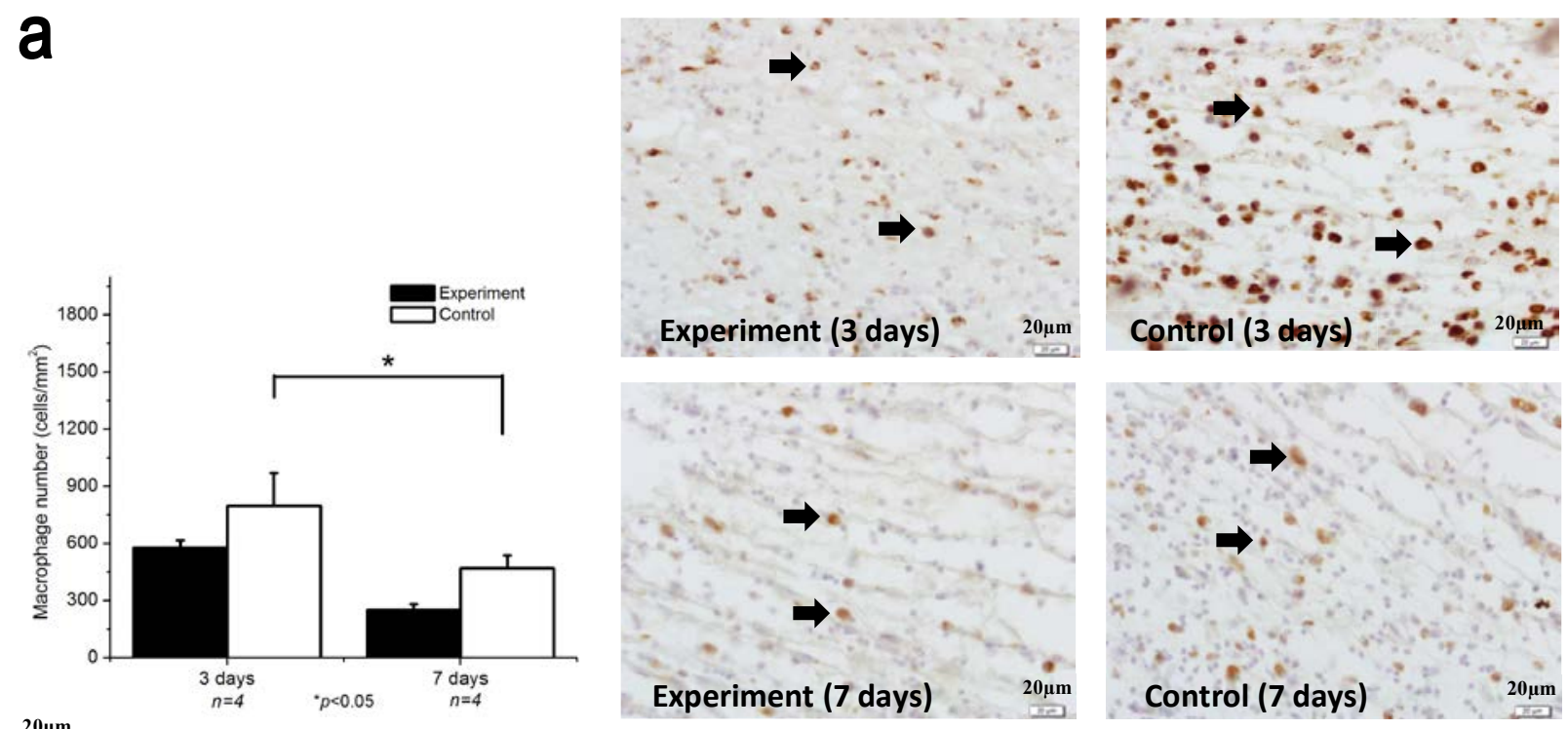

$20 \mu \mathrm{m}$

Figure 7 


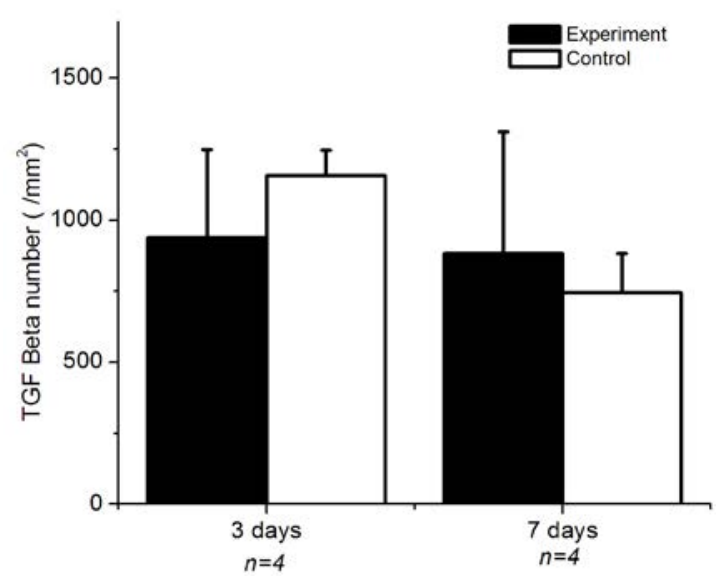

Figure 8 